\title{
OPTIMAL DUAL FUSION FRAMES FOR PROBABILISTIC ERASURES*
}

\author{
PATRICIA MARIELA MORILLAS ${ }^{\dagger}$
}

\begin{abstract}
For any fixed fusion frame, its optimal dual fusion frames for reconstruction is studied in case of erasures of subspaces. It is considered that a probability distribution of erasure of subspaces is given and that a blind reconstruction procedure is used, where the erased data are set to zero. It is proved that there are always optimal duals. Sufficient conditions for the canonical dual fusion frame being either the unique optimal dual, a non-unique optimal dual, or a non optimal dual, are obtained. The reconstruction error is analyzed, using the optimal duals in the probability model considered here and using the optimal duals in a non-probability model.
\end{abstract}

Key words. Frames, Fusion frames, Dual fusion frames, Probabilistic erasures, Optimal dual fusion frames.

AMS subject classifications. 42C15, 15A60, 47N10, 94A12, 68M10.

1. Introduction. Fusion frames (or frames of subspaces) $[3,5]$ for a separable Hilbert space $\mathcal{H}$ are collections of closed subspaces and weights. They are a generalization of frames $[6,13]$ and are applied in distributed processing settings such as distributing sensing, parallel processing and packet encoding. In the present paper we are interested in the application of fusion frames in the transmission of signals when some of the transmitted information is lost. The signals will be vectors of a finite-dimensional Hilbert space.

Given a fusion frame for $\mathcal{H}$, each element $f \in \mathcal{H}$ is represented with packets of coefficients. In applications, some of these packets may be erased and it is necessary performing the reconstruction of $f$ with the available information. One approach to this problem is the study and the construction of optimal fusion frames $[1,4,14,16,17]$. Here optimality is understood as to have yet a fusion frame or as to minimize certain reconstruction error, after the erasure of subspaces. When the fusion frame used for the representation of the elements of $\mathcal{H}$ is fixed, other approaches are needed. The concept of dual fusion frame introduced and studied in $[7,8]$ allows us to analyze how to select optimal dual fusion frames, for this fixed fusion frame, in order to perform the reconstruction.

In [8], the authors studied optimal dual fusion frames for a fixed fusion frame when a blind reconstruction process is used. This is done in a similar way as in [9, 12] for frames and in [15] for projective reconstruction systems. In real implementations the subspaces are generally erased with different probabilities. In the present paper, we address this situation studying optimal dual fusion frames when a probability distribution of erasure of subspaces is given. We consider a probability model in concordance with frames considered in $[10,11]$, but using the Frobenius norm to define the errors as in $[8,15]$. Some of the obtained results can be viewed as analogous ones of those of [10] in the context of dual fusion frames and generalize results of [8] to the probabilistic case.

The outline of the paper is as follows. In Section 2, we review fusion frames and dual fusion frames. In Section 3, we state the mathematical model introduced in [8] for studying optimal dual fusion frames for a

\footnotetext{
* Received by the editors on March 30, 2016. Accepted for publication on May 30, 2017. Handling Editor: Zejun Huang.

${ }^{\dagger}$ Instituto de Matemática Aplicada San Luis, UNSL-CONICET. Ejército de los Andes 950, 5700 San Luis, Argentina (morillas@unsl.edu.ar). Research partially supported by Grants PROIPRO 3-40114 (UNSL), R.3646/14 (CONICET) and PIP 112-201501-00589-CO (CONICET).
} 
fixed fusion frame when a blind reconstruction process is used, considering each of the erased data equal to zero. We introduce a modification to this model for studying the case in which a probability distribution of erasure of subspaces is given. For the error defined by the $\ell_{2}$-norm, called the mean squared error, both models lead to the same optimal duals. In the rest of the paper, we consider the error defined by the $\ell_{\infty}$-norm, called the worst case error. We prove that there are always optimal duals. We also obtain easy verifiable sufficient conditions that assure that the canonical dual fusion frame (the most studied and used dual so far) is either the unique probability optimal dual, a non-unique probability optimal dual, or a non-optimal probability dual. In Section 4, we investigate the convenience of using the probability model for determining optimal duals instead of the non-probability model considered in [8], when the subspaces have not the same probability of erasure.

2. Preliminaries. In this section, we recall the concepts of fusion frame $[3,5]$ (see also [2, Chapter 13]) and dual fusion frame $[7,8]$. We refer to the mentioned works for more details. We begin introducing some notations.

2.1. Notation. Let $\mathcal{H}, \mathcal{K}$ be finite-dimensional Hilbert spaces over $\mathbb{F}=\mathbb{R}$ or $\mathbb{F}=\mathbb{C}$. If $V \subset \mathcal{H}$ is a subspace, $\pi_{V}$ denotes the orthogonal projection onto $V$. Let $L(\mathcal{H}, \mathcal{K})$ be the space of linear transformations from $\mathcal{H}$ to $\mathcal{K}$. Given $T \in L(\mathcal{H}, \mathcal{K})$, we write $R(T)$ and $T^{*}$ to denote the image and the adjoint of $T$, respectively. If $T \in L(\mathcal{H}, \mathcal{K})$ is injective, then $\mathfrak{L}_{T}$ denotes the set of left inverses of $T$.

The inner product and the norm in $\mathcal{H}$ will be denoted by $\langle\cdot, \cdot\rangle$ and $\|\cdot\|$, respectively. If $T \in L(\mathcal{H}, \mathcal{K})$, then $\|T\|$ denotes the Frobenius norm of $T$.

In the sequel, $m, n, d \in \mathbb{N}$, and $\mathcal{H}$ will be a finite-dimensional Hilbert space over $\mathbb{F}$ of dimension $d$. For $p \in \mathbb{N} \cup\{\infty\}$ let $\|\cdot\|_{p}$ denote the $p$-norm in $\mathbb{F}^{n}$.

For a set $A$, let $\chi_{A}: A \rightarrow\{0,1\}$ be the characteristic function of $A$. We abbreviate $\chi_{\{a\}}=\chi_{a}$.

2.2. Fusion frames. The representation of each $f \in \mathcal{H}$ via fusion frames is given by projections onto multidimensional subspaces.

DEFINITION 2.1. Let $\left\{W_{i}\right\}_{i=1}^{m}$ be a family of subspaces of $\mathcal{H}$, and let $\left\{w_{i}\right\}_{i=1}^{m}$ be a family of weights, i.e., $w_{i}>0$ for $i=1, \ldots, m$. Then $\left\{\left(W_{i}, w_{i}\right)\right\}_{i=1}^{m}$ is called a Bessel fusion sequence for $\mathcal{H}$.

We will denote $\left\{W_{i}\right\}_{i=1}^{m}$ with $\mathbf{W},\left\{w_{i}\right\}_{i=1}^{m}$ with $\mathbf{w}$ and $\left\{\left(W_{i}, w_{i}\right)\right\}_{i=1}^{m}$ with $(\mathbf{W}, \mathbf{w})$. If $T \in L(\mathcal{H}, \mathcal{K})$, then we will write $(T \mathbf{W}, \mathbf{w})$ for $\left\{\left(T W_{i}, w_{i}\right)\right\}_{i=1}^{m}$.

Let $\mathcal{W}=\left\{\left(f_{i}\right)_{i=1}^{m}: f_{i} \in W_{i}\right\}$ be the Hilbert space with $\left\langle\left(f_{i}\right)_{i=1}^{m},\left(g_{i}\right)_{i=1}^{m}\right\rangle=\sum_{i=1}^{m}\left\langle f_{i}, g_{i}\right\rangle$.

Definition 2.2. Let $(\mathbf{W}, \mathbf{w})$ be a Bessel fusion sequence.

1. The synthesis operator of $(\mathbf{W}, \mathbf{w})$ is

$$
T_{\mathbf{W}, \mathbf{w}}: \mathcal{W} \rightarrow \mathcal{H}, \quad T_{\mathbf{W}, \mathbf{w}}\left(f_{i}\right)_{i=1}^{m}=\sum_{i=1}^{m} w_{i} f_{i}
$$

The analysis operator is

$$
T_{\mathbf{W}, \mathbf{w}}^{*}: \mathcal{H} \rightarrow \mathcal{W}, \quad T_{\mathbf{W}, \mathbf{w}}^{*} f=\left(w_{i} \pi_{W_{i}}(f)\right)_{i=1}^{m} .
$$

2. $(\mathbf{W}, \mathbf{w})$ is called a fusion frame for $\mathcal{H}$ if $\operatorname{span} \bigcup_{i=1}^{m} W_{i}=\mathcal{H}$, or equivalently, if $R\left(T_{\mathbf{W}, \mathbf{w}}\right)=\mathcal{H}$. 
3. $(\mathbf{W}, \mathbf{w})$ is a Riesz fusion basis if $\mathcal{H}$ is the direct sum of the $W_{i}$.

4. If $(\mathbf{W}, \mathbf{w})$ is a fusion frame for $\mathcal{H}$, the operator

$$
S_{\mathbf{W}, \mathbf{w}}=T_{\mathbf{W}, \mathbf{w}} T_{\mathbf{W}, \mathbf{w}}^{*}: \mathcal{H} \rightarrow \mathcal{H}, \quad S_{\mathbf{W}, \mathbf{w}}(f)=T_{\mathbf{W}, \mathbf{w}} T_{\mathbf{W}, \mathbf{w}}^{*}(f)=\sum_{i=1}^{m} w_{i}^{2} \pi_{W_{i}}(f)
$$

is called the fusion frame operator of $(\mathbf{W}, \mathbf{w})$.

A fusion frame $(\mathbf{W}, \mathbf{w})$ is called an $\alpha$-tight fusion frame if $S_{\mathbf{W}, \mathbf{w}}=\alpha I_{\mathcal{H}}$. If $S_{\mathbf{W}, \mathbf{w}}=I_{\mathcal{H}}$ we say that it is a Parseval fusion frame.

2.3. Dual fusion frames. Next we recall the notion of dual fusion frame $[7,8]$.

Definition 2.3. Let $(\mathbf{W}, \mathbf{w})$ and $(\mathbf{V}, \mathbf{v})$ be two fusion frames for $\mathcal{H}$. Then $(\mathbf{V}, \mathbf{v})$ is a dual fusion frame of $(\mathbf{W}, \mathbf{w})$ if there exists a $Q \in L(\mathcal{W}, \mathcal{V})$ such that

$$
T_{\mathbf{V}, \mathbf{v}} Q T_{\mathbf{W}, \mathbf{w}}^{*}=I_{\mathcal{H}}
$$

If we need to do an explicit reference to the linear transformation $Q$, then we say that $(\mathbf{V}, \mathbf{v})$ is a $Q$-dual fusion frame of $(\mathbf{W}, \mathbf{w})$.

For $J \subseteq\{1, \ldots, m\}$, we consider the selfadjoint operator

$$
M_{J, \mathbf{W}}: \mathcal{W} \rightarrow \mathcal{W}, \quad M_{J, \mathbf{W}}\left(f_{j}\right)_{j=1}^{m}=\left(\chi_{J}(j) f_{j}\right)_{j=1}^{m}
$$

We simply write $M_{J}$ if it is clear to which $\mathbf{W}$ we refer to. We abbreviate $M_{\{j\}, \mathbf{W}}=M_{j, \mathbf{W}}$ and $M_{\{j\}}=M_{j}$.

Definition 2.4. Let $(\mathbf{W}, \mathbf{w})$ and $(\mathbf{V}, \mathbf{v})$ be two Bessel fusion sequences for $\mathcal{H}$ and $Q \in L(\mathcal{W}, \mathcal{V})$.

1. If $Q M_{j, \mathbf{w}} \mathcal{W} \subseteq M_{j, \mathbf{v}} \mathcal{V}$ for each $j \in\{1, \ldots, m\}, Q$ is called block-diagonal.

2. If $Q M_{j, \mathbf{w}} \mathcal{W}=M_{j, \mathbf{v}} \mathcal{V}$ for each $j \in\{1, \ldots, m\}, Q$ is called component preserving.

Observe that $Q$ is block-diagonal if and only if $Q M_{j, \mathbf{w}}=M_{j, \mathbf{v}} Q$ for each $j \in\{1, \ldots, m\}$, or equivalently, $Q M_{J, \mathbf{W}}=M_{J, \mathbf{V}} Q$ for each $J \subseteq\{1, \ldots, m\}$. If $Q$ is block-diagonal, then $Q^{*}$ is block-diagonal. If, in Definition 2.3, $Q$ is block-diagonal (component preserving), then we say that $(\mathbf{V}, \mathbf{v})$ is a block-diagonal dual fusion frame (component preserving dual fusion frame) of $(\mathbf{W}, \mathbf{w})$. In this paper, we restrict us to these types of dual fusion frames.

If $Q$ is block-diagonal, then the reconstruction formula following from (2.1) has the form

$$
f=\sum_{j=1}^{m} v_{j} w_{j} Q_{j} f, \quad \forall f \in \mathcal{H}
$$

where $Q_{j} f:=\left(Q M_{j}\left(\pi_{W_{i}} f\right)_{i=1}^{m}\right)_{j} \in V_{j}$.

The following theorem characterizes the component preserving dual fusion frames of $(\mathbf{W}, \mathbf{w})$ in terms of the left inverses of $T_{\mathbf{W}, \mathbf{w}}^{*}$. Given $A \in L(\mathcal{W}, \mathcal{H})$ and $\mathbf{v}$ a collection of weights, we consider the subspaces $V_{i}=A M_{i} \mathcal{W}$, for each $i=1, \ldots, m$, and $Q_{A, \mathbf{v}}: \mathcal{W} \rightarrow \mathcal{V}, Q_{A, \mathbf{v}}\left(f_{j}\right)_{j=1}^{m}=\left(\frac{1}{v_{i}} A M_{i}\left(f_{j}\right)_{j=1}^{m}\right)_{i=1}^{m}$.

TheOREM 2.5. [8] Let $(\boldsymbol{W}, \mathbf{w})$ be a fusion frame for $\mathcal{H}$. Then $(\mathbf{V}, \mathbf{v})$ is a $Q$-component preserving dual fusion frame of $(\boldsymbol{W}, \mathbf{w})$ if and only if $V_{i}=A M_{i} \mathcal{W}$ for each $i \in\{1, \ldots, m\}$ and $Q=Q_{A, \mathbf{v}}$, for some 
$A \in \mathfrak{L}_{T_{W, \mathbf{w}}^{*}} \cdot$ Moreover, any element of $\mathfrak{L}_{T_{W, \mathbf{w}}^{*}}$ is of the form $T_{\mathbf{V}, \mathbf{v}} Q$ where $(\mathbf{V}, \mathbf{v})$ is some $Q$-component preserving dual fusion frame of $(\boldsymbol{W}, \mathbf{w})$.

By Theorem 2.5, $\left(S_{\mathbf{W}, \mathbf{w}}^{-1} \mathbf{W}, \mathbf{v}\right)$ is a $Q_{A, \mathbf{v}}$-component preserving dual with $A=S_{\mathbf{W}, \mathbf{w}}^{-1} T_{\mathbf{W}, \mathbf{w}}$ and $\mathbf{v}$ a family of arbitrary weights. We refer to this $Q_{S_{\mathbf{W}, \mathbf{w}}^{-1} T \mathbf{W}, \mathbf{w}, \mathbf{v}}$-dual fusion frame as the canonical dual with weights $\mathbf{v}$.

3. Optimal dual fusion frames in a probability model. Let $(\mathbf{W}, \mathbf{w})$ be a fusion frame for $\mathcal{H}$. In applications an element $f \in \mathcal{H}$ (e.g., a signal) is converted into the data vectors $T_{\mathbf{W}, \mathbf{w}}^{*} f=\left(w_{i} \pi_{W_{i}}(f)\right)_{i=1}^{m}$. Sometimes some of the data vectors are lost or erased, and it is necessary to reconstruct $f$ with the information at hand. Let $J \subset\{1, \ldots, m\}$ and suppose that the data vectors corresponding to the subspaces $\left\{W_{j}\right\}_{j \in J}$ are lost. Considering each of the erased data equal to zero, the reconstruction then gives $T_{\mathbf{V}, \mathbf{v}} Q M_{\{1, \ldots, m\} \backslash J} T_{\mathbf{W}, \mathbf{w}}^{*} f$, where $(\mathbf{V}, \mathbf{v})$ is some $Q$-dual of $(\mathbf{W}, \mathbf{w})$. So we need to find those dual fusion frames of $(\mathbf{W}, \mathbf{w})$ that are in some sense optimal for this situation.

Fix $r \in\{1, \ldots, m-1\}$. Let $\mathcal{P}_{r}^{m}:=\{J \subset\{1, \ldots, m\}:|J|=r\}$. Noting that $M_{J}=I_{\mathcal{W}}-M_{\{1, \ldots, m\} \backslash J}$, given a $Q$-block diagonal dual fusion frame $(\mathbf{V}, \mathbf{v})$ of $(\mathbf{W}, \mathbf{w})$ we consider the general vector error

$$
e(r,(\mathbf{W}, \mathbf{w}),(\mathbf{V}, \mathbf{v}), Q)=\left(\left\|T_{\mathbf{V}, \mathbf{v}} Q M_{J} T_{\mathbf{W}, \mathbf{w}}^{*}\right\|\right)_{J \in \mathcal{P}_{r}^{m}} .
$$

We define inductively:

- $e_{1}^{(p)}(\mathbf{W}, \mathbf{w})$ as the infimum of the set of the numbers $\|e(1,(\mathbf{W}, \mathbf{w}),(\mathbf{V}, \mathbf{v}), Q)\|_{p}$ such that $(\mathbf{V}, \mathbf{v})$ is a $Q$-block diagonal dual fusion frame of $(\mathbf{W}, \mathbf{w})$,

- $\mathcal{D}_{1}^{(p)}(\mathbf{W}, \mathbf{w})$ as the set of $((\mathbf{V}, \mathbf{v}), Q)$ where $(\mathbf{V}, \mathbf{v})$ is a $Q$-block diagonal dual fusion frame of $(\mathbf{W}, \mathbf{w})$ and $\|e(1,(\mathbf{W}, \mathbf{w}),(\mathbf{V}, \mathbf{v}), Q)\|_{p}=e_{1}^{(p)}(\mathbf{W}, \mathbf{w})$,

- $e_{r}^{(p)}(\mathbf{W}, \mathbf{w})=\inf \left\{\|e(r,(\mathbf{W}, \mathbf{w}),(\mathbf{V}, \mathbf{v}), Q)\|_{p}:((\mathbf{V}, \mathbf{v}), Q) \in \mathcal{D}_{r-1}^{(p)}(\mathbf{W}, \mathbf{w})\right\}$,

- $\mathcal{D}_{r}^{(p)}(\mathbf{W}, \mathbf{w})=\left\{((\mathbf{V}, \mathbf{v}), Q) \in \mathcal{D}_{r-1}^{(p)}(\mathbf{W}, \mathbf{w}):\|e(r,(\mathbf{W}, \mathbf{w}),(\mathbf{V}, \mathbf{v}), Q)\|_{p}=e_{r}^{(p)}(\mathbf{W}, \mathbf{w})\right\}$,

in case each $\mathcal{D}_{r}^{(p)}(\mathbf{W}, \mathbf{w})$ is non-empty. Each element of $\mathcal{D}_{r}^{(p)}(\mathbf{W}, \mathbf{w})$ will be called a general $(r, p)$-loss optimal dual fusion frame of $(\mathbf{W}, \mathbf{w})$.

3.1. The probability model. Now we are going to suppose that the subspaces of the fusion frame $(\mathbf{W}, \mathbf{w})$ can be lost or erased with non necessarily equal probability. We consider that the probability $p_{i}$ of the erasure for the $i$ th subspace for each $i=1, \ldots, m$ is given. These numbers define a probability distribution of erasure of the subspaces, or equivalently, of the data vectors $T_{\mathbf{W}, \mathbf{w}}^{*} f=\left(w_{i} \pi_{W_{i}}(f)\right)_{i=1}^{m}$ for each $f \in \mathcal{H}$. Clearly we have $\sum_{i=1}^{m} p_{i}=1$ and $0 \leq p_{i} \leq 1$ for each $i=1, \ldots, m$.

In order to define the mathematical model, we consider numbers $q_{i}$ for $i=1, \ldots, m$ such that there exists a strictly increasing real function $F$ with $q_{i}=F\left(p_{i}\right)>0$ for $i=1, \ldots, m$. An advantage of introducing these numbers is that we have $q_{i}>0$ for each $i=1, \ldots, m$ whereas some of the probabilities $p_{i}$ can be equal to zero. A more precise definition of the numbers $q_{i}$ in function of the probabilities $p_{i}$ can be given according with the problem we are studying (see Example 4.5 and for the case of frames see $[10,11]$ ).

We consider the selfadjoint operators $\widehat{M}_{i, \mathbf{w}}: \mathcal{W} \rightarrow \mathcal{W}, \widehat{M}_{i, \mathbf{w}}=q_{i} M_{i, \mathbf{w}}$ and $\widehat{M}_{J, \mathbf{w}}=\sum_{i \in J} \widehat{M}_{i, \mathbf{w}}$. We simply write $\widehat{M}_{i}$ or $\widehat{M}_{J}$ if it is clear to which $\mathbf{W}$ we refer to.

Given a $Q$-block diagonal dual fusion frame $(\mathbf{V}, \mathbf{v})$ of $(\mathbf{W}, \mathbf{w})$, we consider the probability vector error

$$
\widehat{e}(r,(\mathbf{W}, \mathbf{w}),(\mathbf{V}, \mathbf{v}), Q)=\left(\left\|T_{\mathbf{V}, \mathbf{v}} Q \widehat{M}_{J} T_{\mathbf{W}, \mathbf{w}}^{*}\right\|\right)_{J \in \mathcal{P}_{r}^{m}}
$$


We define inductively:

- $\widehat{e}_{1}^{(p)}(\mathbf{W}, \mathbf{w})$ as the infimum of the set of the numbers $\|\widehat{e}(1,(\mathbf{W}, \mathbf{w}),(\mathbf{V}, \mathbf{v}), Q)\|_{p}$ such that $(\mathbf{V}, \mathbf{v})$ is a $Q$-block diagonal dual fusion frame of $(\mathbf{W}, \mathbf{w})$,

- $\widehat{\mathcal{D}}_{1}^{(p)}(\mathbf{W}, \mathbf{w})$ as the set of $((\mathbf{V}, \mathbf{v}), Q)$ where $(\mathbf{V}, \mathbf{v})$ is a $Q$-block diagonal dual fusion frame of $(\mathbf{W}, \mathbf{w})$ and $\|\widehat{e}(1,(\mathbf{W}, \mathbf{w}),(\mathbf{V}, \mathbf{v}), Q)\|_{p}=\widehat{e}_{1}^{(p)}(\mathbf{W}, \mathbf{w})$,

- $\widehat{e}_{r}^{(p)}(\mathbf{W}, \mathbf{w})=\inf \left\{\|\widehat{e}(r,(\mathbf{W}, \mathbf{w}),(\mathbf{V}, \mathbf{v}), Q)\|_{p}:((\mathbf{V}, \mathbf{v}), Q) \in \widehat{\mathcal{D}}_{r-1}^{(p)}(\mathbf{W}, \mathbf{w})\right\}$,

- $\widehat{\mathcal{D}}_{r}^{(p)}(\mathbf{W}, \mathbf{w})=\left\{((\mathbf{V}, \mathbf{v}), Q) \in \widehat{\mathcal{D}}_{r-1}^{(p)}(\mathbf{W}, \mathbf{w}):\|\widehat{e}(r,(\mathbf{W}, \mathbf{w}),(\mathbf{V}, \mathbf{v}), Q)\|_{p}=\widehat{e}_{r}^{(p)}(\mathbf{W}, \mathbf{w})\right\}$,

in case each $\widehat{\mathcal{D}}_{r}^{(p)}(\mathbf{W}, \mathbf{w})$ is non-empty. Each element of $\widehat{\mathcal{D}}_{r}^{(p)}(\mathbf{W}, \mathbf{w})$ will be called a probability $(r, p)$-loss optimal dual fusion frame of $(\mathbf{W}, \mathbf{w})$.

\subsection{Probability optimal dual fusion frames. Note that}

$$
M_{i} T_{\mathbf{W}, \mathbf{w}}^{*} T_{\mathbf{W}, \mathbf{w}} M_{i}^{*}=w_{i}^{2} M_{i}
$$

Let $A \in L(\mathcal{W}, \mathcal{H})$. Then

$$
\left\|A \widehat{M}_{i} T_{\mathbf{W}, \mathbf{w}}^{*}\right\|^{2}=q_{i}^{2} w_{i}^{2}\left\|A M_{i}\right\|^{2}
$$

We also have

$$
\left\|S_{\mathbf{W}, \mathbf{w}}^{-1} T_{\mathbf{W}, \mathbf{w}} M_{i}\right\|=w_{i}\left\|S_{\mathbf{W}, \mathbf{w}}^{-1} \pi_{W_{i}}\right\|
$$

For $p=2$ we obtain the probability mean squared error $\|\widehat{e}(r,(\mathbf{W}, \mathbf{w}),(\mathbf{V}, \mathbf{v}), Q)\|_{2}$. In this case, probability optimal duals coincide with general optimal duals, i.e., $\widehat{\mathcal{D}}_{r}^{(2)}(\mathbf{W}, \mathbf{w})=\mathcal{D}_{r}^{(2)}(\mathbf{W}, \mathbf{w})$ for each $r \geq 1$. Specifically, Theorem 5.1 in [8], with a similar proof, remains valid for probability $(r, 2)$-loss optimal component preserving dual fusion frames.

In what follows, we consider $p=\infty$. In this case, we obtain the probability worst-case error,

$$
\|\widehat{e}(r,(\mathbf{W}, \mathbf{w}),(\mathbf{V}, \mathbf{v}), Q)\|_{\infty}=\max _{J \in \mathcal{P}_{r}^{m}}\left\|T_{\mathbf{V}, \mathbf{v}} Q \widehat{M}_{J, \mathbf{W}} T_{\mathbf{W}, \mathbf{w}}^{*}\right\|
$$

In the sequel, we restrict us to component preserving dual fusion frames. Note that by [8, Remark 3.6], we can always replace a block-diagonal dual fusion frame with a component preserving dual fusion frame that has the same general (or probability) vector error.

Some important properties of the set $\widehat{\mathcal{D}}_{r}^{(\infty)}(\mathbf{W}, \mathbf{w})$ are given in the following theorem.

THEOREM 3.1. Let $(\mathbf{W}, \mathbf{w})$ be a fusion frame for $\mathcal{H}$. Then $\widehat{\mathcal{D}}_{r}^{(\infty)}(\mathbf{W}, \mathbf{w})$ is non-empty, compact and convex for each $r=1, \ldots, m-1$.

Proof. The map $\|.\|_{\mathbf{W}, \mathbf{w}}^{(r)}: L(\mathcal{W}, \mathcal{H}) \rightarrow \mathbb{R}^{+},\|A\|_{\mathbf{W}, \mathbf{w}}^{(r)}=\max _{J \in \mathcal{P}_{r}^{m}}\left\|A \widehat{M}_{J} T_{\mathbf{W}, \mathbf{w}}^{*}\right\|$, is a norm in $L(\mathcal{W}, \mathcal{H})$. To see this, let $A \in L(\mathcal{W}, \mathcal{H})$ such that $\|A\|_{\mathbf{W}, \mathbf{w}}^{(r)}=0$. So, $A \widehat{M}_{J} T_{\mathbf{W}, \mathbf{w}}^{*}=0$ for each $J \in \mathcal{P}_{r}^{m}$. If $r \geq 2$, let $k, k^{\prime} \in\{1, \ldots, m\}, k \neq k^{\prime}$ and $J^{\prime} \subseteq\{1, \ldots, m\} \backslash\left\{k, k^{\prime}\right\}$ such that $\left|J^{\prime}\right|=r-1$. We have $A \widehat{M}_{k} T$ W.w $=$ $A \widehat{M}_{k^{\prime}} T_{\mathbf{W}, \mathbf{w}}^{*}=-A \widehat{M}_{J^{\prime}} T_{\mathbf{W}, \mathbf{w}}^{*}$. This shows that, $A \widehat{M}_{J} T_{\mathbf{W}, \mathbf{w}}^{*}=r A \widehat{M}_{k} T_{\mathbf{W}, \mathbf{w}}^{*}$, and consequently, $A \widehat{M}_{k} T T_{\mathbf{W}, \mathbf{w}}^{*}=0$ for each $k \in J$ and each $J \in \mathcal{P}_{r}^{m}$. By (3.2) this implies that $A M_{k}=0$ for $k=1, \ldots, m$, and thus $A=\sum_{k=1}^{m} A M_{k}=0$. The other norm properties are immediate.

Since the set $\mathfrak{L}_{T_{\mathbf{W}, \mathbf{w}}^{*}}$ is closed in $L(\mathcal{W}, \mathcal{H})$ under the usual norm and all norms in a finite-dimensional Hilbert space are equivalent, $\mathfrak{L}_{T_{\mathbf{W}, \mathbf{w}}^{*}}$ is a closed subset of $L(\mathcal{W}, \mathcal{H})$ under the norm $\|\cdot\|_{\mathbf{W}, \mathbf{w}}^{(1)}$. Given $B_{0} \in \mathfrak{L}_{T_{\mathbf{W}, \mathbf{w}}^{*}}$, 
$B_{0} \neq 0$, there exists an $A_{0}$ in the non-empty compact set $\left\{A \in \mathfrak{L}_{T_{\mathbf{W}, \mathbf{w}}^{*}}:\|A\|_{\mathbf{W}, \mathbf{w}}^{(1)} \leq\left\|B_{0}\right\|_{\mathbf{W}, \mathbf{w}}^{(1)}\right\}$ where the continuous map $\|.\|_{\mathbf{W}, \mathbf{w}}^{(1)}$ attains its minimum. So, $\left\|A_{0}\right\|_{\mathbf{W}, \mathbf{w}}^{(1)}=\min _{A \in \mathfrak{L}_{T_{\mathbf{W}, \mathbf{w}}^{*}}}\|A\|_{\mathbf{W}, \mathbf{w}}^{(1)}$, and the set

$$
\left\{A \in \mathfrak{L}_{T_{\mathbf{W}, \mathbf{w}}^{*}}: \max _{1 \leq i \leq m}\left\|A \widehat{M}_{i} T_{\mathbf{W}, \mathbf{w}}^{*}\right\|=\min _{B \in \mathfrak{L}_{T_{\mathbf{W}, \mathbf{w}}^{*}}} \max _{1 \leq i \leq m}\left\|B \widehat{M}_{i} T_{\mathbf{W}, \mathbf{w}}^{*}\right\|\right\}
$$

is non-empty and compact. Since $\mathfrak{L}_{T_{\mathbf{W}, \mathbf{w}}^{*}}$ is convex and $\|\cdot\|_{\mathbf{W}, \mathbf{w}}^{(1)}$ is a convex map, the set (3.4) is convex. Consequently, by Theorem $2.5, \widehat{\mathcal{D}}_{1}^{(\infty)}(\mathbf{W}, \mathbf{w})$ is non-empty, compact and convex.

An inductive argument shows that $\widehat{\mathcal{D}}_{r}^{(\infty)}(\mathbf{W}, \mathbf{w})$ is non-empty compact and convex for each $r \geq 2$.

By Theorem 2.5, if $(\mathbf{W}, \mathbf{w})$ is a fusion frame for $\mathcal{H}$ then $A$ is an element of the set (3.4) if and only if the $Q_{A, \mathbf{v}}$-component preserving dual fusion frames with arbitrary vector of weights $\mathbf{v},\left\{\left(A M_{i} \mathcal{W}, v_{i}\right)\right\}_{i=1}^{m}$, are probability $(1, \infty)$-loss optimal component preserving dual fusion frames of $(\mathbf{W}, \mathbf{w})$. Using [8, Lemma 3.4] we also have:

Lemma 3.2. Let $(\mathbf{W}, \mathbf{w})$ be a fusion frame for $\mathcal{H}$. Then $S_{\mathbf{W}, \mathbf{w}}^{-1} T_{\mathbf{W}, \mathbf{w}}$ is the unique element of the set (3.4) if and only if the canonical dual fusion frames $\left(S_{\mathbf{W}, \mathbf{w}}^{-1} \mathbf{W}, \mathbf{v}\right)$, with arbitrary vector of weights $\mathbf{v}$, are the unique probability $(1, \infty)$-loss optimal component preserving dual fusion frames of $(\mathbf{W}, \mathbf{w})$.

The next theorem gives sufficient conditions that assure that the only probability $(r, \infty)$-loss optimal component preserving dual fusion frames are the canonical ones.

Theorem 3.3. Let $(\mathbf{W}, \mathbf{w})$ be a fusion frame for $\mathcal{H}, c=\max \left\{q_{i} w_{i}^{2}\left\|S_{\mathbf{W}, \mathbf{w}}^{-1} \pi_{W_{i}}\right\|: i \in\{1, \ldots, m\}\right\}$, $\Lambda_{1}=\left\{i \in\{1, \ldots, m\}: q_{i} w_{i}^{2}\left\|S_{\mathbf{W}, \mathbf{w}}^{-1} \pi_{W_{i}}\right\|=c\right\}, \Lambda_{2}=\{1, \ldots, m\} \backslash \Lambda_{1}$ and $\mathcal{H}_{j}=\operatorname{span} \bigcup_{i \in \Lambda_{j}} W_{i}, j=1,2$. If $\mathcal{H}_{1} \cap \mathcal{H}_{2}=\{0\}$ and $\left\{\left(W_{i}, w_{i}\right)\right\}_{i \in \Lambda_{2}}$ is a Riesz fusion basis for $\mathcal{H}_{2}$, then the only probability $(r, \infty)$-loss optimal component preserving dual fusion frames of $(\mathbf{W}, \mathbf{w})$ are the canonical ones $\left(S_{\mathbf{W}, \mathbf{w}}^{-1} \mathbf{W}, \mathbf{v}\right)$ with arbitrary vector of weights $\mathbf{v}$.

Proof. By Theorem 3.1 and Theorem 2.5, there exists $A \in \mathfrak{L}_{T_{\mathbf{W}, \mathbf{w}}^{*}}$ such that

$$
\max _{1 \leq i \leq m}\left\|A \widehat{M}_{i} T_{\mathbf{W}, \mathbf{w}}^{*}\right\|=\min _{B \in \mathfrak{L}_{T_{\mathbf{W}, \mathbf{w}}^{*}}} \max _{1 \leq i \leq m}\left\|B \widehat{M}_{i} T_{\mathbf{W}, \mathbf{w}}^{*}\right\|
$$

So,

$$
\max _{1 \leq i \leq m}\left\|A \widehat{M}_{i} T_{\mathbf{W}, \mathbf{w}}^{*}\right\| \leq \max _{1 \leq i \leq m}\left\|S_{\mathbf{W}, \mathbf{w}}^{-1} T_{\mathbf{W}, \mathbf{w}} \widehat{M}_{i} T_{\mathbf{W}, \mathbf{w}}^{*}\right\| .
$$

and then, by (3.2), (3.3) and hypothesis,

$$
\left\|A M_{i}\right\| \leq\left\|S_{\mathbf{W}, \mathbf{w}}^{-1} T_{\mathbf{W}, \mathbf{w}} M_{i}\right\|, \quad \forall i \in \Lambda_{1} .
$$

Since $A T_{\mathbf{W}, \mathbf{w}}^{*}=S_{\mathbf{W}, \mathbf{w}}^{-1} T_{\mathbf{W}, \mathbf{w}} T_{\mathbf{W}, \mathbf{w}}^{*}=I_{\mathcal{H}}, T_{\mathbf{W}, \mathbf{w}}\left(A-S_{\mathbf{W}, \mathbf{w}}^{-1} T_{\mathbf{W}, \mathbf{w}}\right)^{*}=0$. By hypothesis this implies that

$$
T_{\mathbf{W}, \mathbf{w}} M_{\Lambda_{1}}\left(A-S_{\mathbf{W}, \mathbf{w}}^{-1} T_{\mathbf{W}, \mathbf{w}}\right)^{*} f=\sum_{i \in \Lambda_{1}} w_{i}\left(\left(A-S_{\mathbf{W}, \mathbf{w}}^{-1} T_{\mathbf{W}, \mathbf{w}}\right)^{*} f\right)_{i}=0
$$

for all $f \in \mathcal{H}$ and $\left(\left(A-S_{\mathbf{W}, \mathbf{w}}^{-1} T_{\mathbf{W}, \mathbf{w}}\right)^{*} f\right)_{i}=0$ for each $i \in \Lambda_{2}$ and for each $f \in \mathcal{H}$, or equivalently, $M_{\Lambda_{2}}\left(A-S_{\mathbf{W}, \mathbf{w}}^{-1} T_{\mathbf{W}, \mathbf{w}}\right)^{*}=0$, i.e.,

$$
A M_{\Lambda_{2}}=S_{\mathbf{W}, \mathbf{w}}^{-1} T_{\mathbf{W}, \mathbf{w}} M_{\Lambda_{2}} .
$$


Using

$$
\begin{aligned}
\left\|A M_{i}\right\|^{2}= & \left\|S_{\mathbf{W}, \mathbf{w}}^{-1} T_{\mathbf{W}, \mathbf{w}} M_{i}\right\|^{2}+\left\|\left(A-S_{\mathbf{W}, \mathbf{w}}^{-1} T_{\mathbf{W}, \mathbf{w}}\right) M_{i}\right\|^{2} \\
& +2 \operatorname{Re}\left(\operatorname{tr}\left[\left(A-S_{\mathbf{W}, \mathbf{w}}^{-1} T_{\mathbf{W}, \mathbf{w}}\right) M_{i} T_{\mathbf{W}, \mathbf{w}}^{*} S_{\mathbf{W}, \mathbf{w}}^{-1}\right]\right),
\end{aligned}
$$

by $(3.5)$,

$$
\left\|\left(A-S_{\mathbf{W}, \mathbf{w}}^{-1} T_{\mathbf{W}, \mathbf{w}}\right) M_{i}\right\|^{2}+2 \operatorname{Re}\left(\operatorname{tr}\left[\left(A-S_{\mathbf{W}, \mathbf{w}}^{-1} T_{\mathbf{W}, \mathbf{w}}\right) M_{i} T_{\mathbf{W}, \mathbf{w}}^{*} S_{\mathbf{W}, \mathbf{w}}^{-1}\right]\right) \leq 0
$$

for all $i \in \Lambda_{1}$. By $(3.6),\left(A-S_{\mathbf{W}, \mathbf{w}}^{-1} T_{\mathbf{W}, \mathbf{w}}\right) M_{\Lambda_{1}} T_{\mathbf{W}, \mathbf{w}}^{*}=0$, so

$$
\begin{aligned}
\sum_{i \in \Lambda_{1}} \operatorname{tr}\left[\left(A-S_{\mathbf{W}, \mathbf{w}}^{-1} T_{\mathbf{W}, \mathbf{w}}\right) M_{i} T_{\mathbf{W}, \mathbf{w}}^{*} S_{\mathbf{W}, \mathbf{w}}^{-1}\right] & =\operatorname{tr}\left[\left(A-S_{\mathbf{W}, \mathbf{w}}^{-1} T_{\mathbf{W}, \mathbf{w}}\right) M_{\Lambda_{1}} T_{\mathbf{W}, \mathbf{w}}^{*} S_{\mathbf{W}, \mathbf{w}}^{-1}\right] \\
& =0 .
\end{aligned}
$$

Thus, by (3.8), $\sum_{i \in \Lambda_{1}}\left\|\left(A-S_{\mathbf{W}, \mathbf{w}}^{-1} T_{\mathbf{W}, \mathbf{w}}\right) M_{i}\right\|^{2} \leq 0$, and consequently,

$$
A M_{i}=S_{\mathbf{W}, \mathbf{w}}^{-1} T_{\mathbf{W}, \mathbf{w}} M_{i}, \quad \forall i \in \Lambda_{1}
$$

This jointly with (3.7) shows that $A=S_{\mathbf{W}, \mathbf{w}}^{-1} T_{\mathbf{W}, \mathbf{w}}$. Thus $S_{\mathbf{W}, \mathbf{w}}^{-1} T_{\mathbf{W}, \mathbf{w}}$ is the unique element of the set (3.4), and the conclusion follows from Lemma 3.2 and the inductive definition of probability $(r, \infty)$-loss optimal dual fusion frames.

The following corollary can be deduced easily from Theorem 3.3.

Corollary 3.4. Let $(\mathbf{W}, \mathbf{w})$ be a fusion frame for $\mathcal{H}$. If $q_{i} w_{i}^{2}\left\|S_{\mathbf{W}, \mathbf{w}}^{-1} \pi_{W_{i}}\right\|=$ c for each $i=1, \ldots, m$, then the only probability $(r, \infty)$-loss optimal component preserving dual fusion frames of $(\mathbf{W}, \mathbf{w})$ are the canonical ones $\left(S_{\mathbf{W}, \mathbf{w}}^{-1} \mathbf{W}, \mathbf{v}\right)$ with arbitrary vector of weights $\mathbf{v}$.

For tight fusion frames we have:

COROllary 3.5. Let $(\mathbf{W}, \mathbf{w})$ be an $\alpha$-tight fusion frame for $\mathcal{H}$. If $q_{i} w_{i}^{2} \sqrt{\operatorname{dim}\left(W_{i}\right)}=c$ for each $i=$ $1, \ldots, m$, then the only probability $(r, \infty)$-loss optimal component preserving dual fusion frames of $(\mathbf{W}, \mathbf{w})$ are the canonical ones $(\mathbf{W}, \mathbf{v})$ with arbitrary vector of weights $\boldsymbol{v}$.

Proof. By hypothesis, $q_{i} w_{i}^{2}\left\|S_{\mathbf{W}, \mathbf{w}}^{-1} \pi_{W_{i}}\right\|=\frac{q_{i} w_{i}^{2}}{\alpha} \operatorname{dim}\left(W_{i}\right)^{1 / 2}=\frac{c}{\alpha}$ for each $i=1, \ldots, m$, so the proof follows from the previous corollary.

The next two propositions can be used to know whether a given fusion frame has not the canonical duals as the unique $(1, \infty)$-loss optimal component preserving dual fusion frames. They are converses of Theorem 3.3.

Proposition 3.6. Let $(\mathbf{W}, \mathbf{w})$ be a fusion frame for $\mathcal{H}$. Let $c, \Lambda_{j}$ and $\mathcal{H}_{j}, j=1,2$, as in Theorem 3.3. If the only probability $(1, \infty)$-loss optimal component preserving dual fusion frames of $(\mathbf{W}, \mathbf{w})$ are the canonical ones $\left(S_{\mathbf{W}, \mathbf{w}}^{-1} \mathbf{W}, \mathbf{v}\right)$ with arbitrary vector of weights $\mathbf{v}$, then $\left\{\left(W_{i}, w_{i}\right)\right\}_{i \in \Lambda_{2}}$ is a Riesz fusion basis for $\mathcal{H}_{2}$.

Proof. We first note that the Bessel sequence $\left\{\left(W_{i}, w_{i}\right)\right\}_{i \in \Lambda_{2}}$ is not a Riesz fusion basis for $\mathcal{H}_{2}$ if and only if the corresponding analysis operator has more than one inverse.

Therefore, if $\left\{\left(W_{i}, w_{i}\right)\right\}_{i \in \Lambda_{2}}$ is not a Riesz fusion basis for $\mathcal{H}_{2}$ there exists $R \in L(\mathcal{W}, \mathcal{H})$ such that $R M_{i}=0$ for each $i \in \Lambda_{1}, R M_{\Lambda_{2}} \neq 0$ and $R M_{\Lambda_{2}} T_{\mathbf{W}, \mathbf{w}}^{*}=0$. If $t \in \mathbb{R}, S_{\mathbf{W}, \mathbf{w}}^{-1} T_{\mathbf{W}, \mathbf{w}}+t R \in \mathfrak{L}_{T_{\mathbf{W}, \mathbf{w}}^{*}}$ and, by $(3.2)$ and (3.3),

$$
\left\|\left(S_{\mathbf{W}, \mathbf{w}}^{-1} T_{\mathbf{W}, \mathbf{w}}+t R\right) \widehat{M}_{i} T_{\mathbf{W}, \mathbf{w}}^{*}\right\|=q_{i} w_{i}^{2}\left\|S_{\mathbf{W}, \mathbf{w}}^{-1} \pi_{W_{i}}\right\|=c
$$


for each $i \in \Lambda_{1}$, and if $|t|$ is small enough, then

$$
\left\|\left(S_{\mathbf{W}, \mathbf{w}}^{-1} T_{\mathbf{W}, \mathbf{w}}+t R\right) \widehat{M}_{i} T_{\mathbf{W}, \mathbf{w}}^{*}\right\| \leq q_{i} w_{i}^{2}\left\|S_{\mathbf{W}, \mathbf{w}}^{-1} \pi_{W_{i}}\right\|+|t|\left\|R \widehat{M}_{i} T_{\mathbf{W}, \mathbf{w}}^{*}\right\|<c
$$

for each $i \in \Lambda_{2}$. This shows that $S_{\mathbf{W}, \mathbf{w}}^{-1} T_{\mathbf{W}, \mathbf{w}}+t R$ with $t \neq 0$ and $|t|$ small enough is an element of the set (3.4), which, by Lemma 3.2, contradicts the hypothesis. Thus, $\left\{\left(W_{i}, w_{i}\right)\right\}_{i \in \Lambda_{2}}$ is a Riesz fusion basis for $\mathcal{H}_{2} . \square$

Proposition 3.7. Let $(\mathbf{W}, \mathbf{w})$ be a fusion frame for $\mathcal{H}$. Let $c, \Lambda_{j}$ and $\mathcal{H}_{j}, j=1,2$, as in Theorem 3.3 . Suppose that there exists $\Lambda_{1}^{\prime} \subseteq \Lambda_{1}$ such that $\left\{\left(W_{i}, w_{i}\right)\right\}_{i \in \Lambda_{1}^{\prime}}$ is a Riesz fusion basis for $\mathcal{H}_{1}$. If the only probability $(1, \infty)$-loss optimal component preserving dual fusion frames of $(\mathbf{W}, \mathbf{w})$ are the canonical ones $\left(S_{\mathbf{W}, \mathbf{w}}^{-1} \mathbf{W}, \mathbf{v}\right)$ with arbitrary vector of weights $\mathbf{v}$, then $\mathcal{H}_{1} \cap \mathcal{H}_{2}=\{0\}$.

Proof. Suppose that $\mathcal{H}_{1} \cap \mathcal{H}_{2} \neq\{0\}$. This implies that there exists $\Lambda_{1}^{\prime \prime} \subseteq \Lambda_{1}^{\prime}$ and $\left(f_{j}\right)_{j=1}^{m} \in M_{\Lambda_{1}^{\prime \prime} \cup \Lambda_{2}} \mathcal{W}$ such that $f_{j} \neq 0$ for each $j \in \Lambda_{1}^{\prime \prime}$ and $T_{\mathbf{W}, \mathbf{w}}\left(f_{j}\right)_{j=1}^{m}=0$.

Let $\left\{e_{i, l}\right\}_{l \in L_{i}}$ be an orthonormal basis for $W_{i}$ for each $i=1, \ldots, m$. Then, by hypothesis, $\left\{S_{\mathbf{W}, \mathbf{w}}^{-1} e_{i, l}\right\}_{i \in \Lambda_{1}^{\prime \prime}, l \in L_{i}}$ is linearly independent. Hence, by [6, Theorem 6.5.1], there exists $f \in \mathcal{H}$ such that $\left\langle f, S_{\mathbf{W}, \mathbf{w}}^{-1} e_{i, l}\right\rangle=-\left\langle f_{i}, e_{i, l}\right\rangle$ for each $i \in \Lambda_{1}^{\prime \prime}$ and $l \in L_{i}$.

Let $R \in L(\mathcal{W}, \mathcal{H})$, given by $R\left(g_{j}\right)_{j=1}^{m}=\left\langle\left(g_{j}\right)_{j=1}^{m},\left(f_{j}\right)_{j=1}^{m}\right\rangle f$ for each $\left(g_{j}\right)_{j=1}^{m} \in \mathcal{W}$. We have $R M_{k}\left(g_{j}\right)_{j=1}^{m}=$ $\left\langle g_{k}, f_{k}\right\rangle f$ if $k \in \Lambda_{1}^{\prime \prime} \cup \Lambda_{2}$ and $R M_{k}\left(g_{j}\right)_{j=1}^{m}=0$ if $k \notin \Lambda_{1}^{\prime \prime} \cup \Lambda_{2}$. We have $R^{*}: \mathcal{H} \rightarrow \mathcal{W}, R^{*} g=\langle g, f\rangle\left(f_{j}\right)_{j=1}^{m}$.

Since $T_{\mathbf{W}, \mathbf{w}} R^{*}=0$, then $S_{\mathbf{W}, \mathbf{w}}^{-1} T_{\mathbf{W}, \mathbf{w}}+t R \in \mathfrak{L}_{T_{\mathbf{W}, \mathbf{w}}^{*}}$ for each $t \in \mathbb{R}$.

If $k \notin \Lambda_{1}^{\prime \prime} \cup \Lambda_{2}$, , by $(3.2)$ and (3.3),

$$
\left\|\left(S_{\mathbf{W}, \mathbf{w}}^{-1} T_{\mathbf{W}, \mathbf{w}}+t R\right) \widehat{M}_{k} T_{\mathbf{W}, \mathbf{w}}^{*}\right\|^{2}=q_{i}^{2} w_{i}^{4}\left\|S_{\mathbf{W}, \mathbf{w}}^{-1} \pi_{W_{i}}\right\|^{2}=c^{2} .
$$

Taking into account that $\left\{\left\{\left(\chi_{j}(i) e_{i, l}\right)_{i=1}^{m}\right\}_{l \in L_{j}}\right\}_{j=1}^{m}$ is an orthonormal basis for $\mathcal{W}$, if $k \in \Lambda_{1}^{\prime \prime}$,

$$
\begin{aligned}
\operatorname{tr}\left(T_{\mathbf{W}, \mathbf{w}}^{*} S_{\mathbf{W}, \mathbf{w}}^{-1} R M_{k}\right) & =\sum_{j=1}^{m} \sum_{l_{j} \in L_{j}}\left\langle T_{\mathbf{W}, \mathbf{w}}^{*} S_{\mathbf{W}, \mathbf{w}}^{-1} R M_{k}\left(\chi_{j}(i) e_{i, l_{j}}\right)_{i=1}^{m},\left(\chi_{j}(i) e_{i, l_{j}}\right)_{i=1}^{m}\right\rangle \\
& =\sum_{l \in L_{k}}\left\langle R M_{k}\left(\chi_{k}(i) e_{i, l}\right)_{i=1}^{m}, w_{k} S_{\mathbf{W}, \mathbf{w}}^{-1} e_{k, l}\right\rangle \\
& =w_{k} \sum_{l \in L_{k}}\left\langle\left\langle e_{k, l}, f_{k}\right\rangle f, S_{\mathbf{W}, \mathbf{w}}^{-1} e_{k, l}\right\rangle \\
& =-w_{k} \sum_{l \in L_{k}}\left|\left\langle f_{k}, e_{k, l}\right\rangle\right|^{2}=-w_{k}\left\|f_{k}\right\|^{2}<0 .
\end{aligned}
$$

Hence, by (3.2) and (3.3), if $t>0$ is small enough,

$$
\left\|\left(S_{\mathbf{W}, \mathbf{w}}^{-1} T_{\mathbf{W}, \mathbf{w}}+t R\right) \widehat{M}_{k} T_{\mathbf{W}, \mathbf{w}}^{*}\right\|^{2}=q_{k}^{2} w_{k}^{4}\left\|S_{\mathbf{W}, \mathbf{w}}^{-1} \pi_{W_{k}}\right\|^{2}+t^{2} q_{k}^{2} w_{k}^{2}\left\|R M_{k}\right\|^{2}+2 t q_{k}^{2} w_{k}^{2} \operatorname{Re}\left(\operatorname{tr}\left(T_{\mathbf{W}, \mathbf{w}}^{*} S_{\mathbf{W}, \mathbf{w}}^{-1} R M_{k}\right)\right)<c^{2}
$$

for each $k \in \Lambda_{1}^{\prime \prime}$, and

$$
\left\|\left(S_{\mathbf{W}, \mathbf{w}}^{-1} T_{\mathbf{W}, \mathbf{w}}+t R\right) \widehat{M}_{k} T_{\mathbf{W}, \mathbf{w}}^{*}\right\| \leq q_{k} w_{k}^{2}\left\|S_{\mathbf{W}, \mathbf{w}}^{-1} \pi_{W_{k}}\right\|+t q_{k} w_{k}\left\|R M_{k}\right\|<c
$$

for each $k \in \Lambda_{2}$. 
This shows that $S_{\mathbf{W}, \mathbf{w}}^{-1} T_{\mathbf{W}, \mathbf{w}}$ is not the only element of the set (3.4) and, by Lemma 3.2, the canonical dual fusion frames $\left(S_{\mathbf{W}, \mathbf{w}}^{-1} \mathbf{W}, \mathbf{v}\right)$ with any vector of weights $\mathbf{v}$ are not the only probability $(1, \infty)$-loss optimal component preserving dual fusion frame of $(\mathbf{W}, \mathbf{w})$. This contradicts the hypothesis, consequently, $\mathcal{H}_{1} \cap \mathcal{H}_{2}=$ $\{0\}$.

The following three theorems describe classes of fusion frames for which the canonical duals are optimal but not the unique ones or are not optimal.

TheOREm 3.8. Let $(\mathbf{W}, \mathbf{w})$ be a fusion frame for $\mathcal{H}$ that is not a Riesz fusion basis. Let $c, \Lambda_{j}$ and $\mathcal{H}_{j}$, $j=1,2$, as in Theorem 3.3. If $\left\{\left(W_{i}, w_{i}\right)\right\}_{i \in \Lambda_{1}}$ is a Riesz fusion basis for $\mathcal{H}_{1}, \Lambda_{2} \neq \emptyset$ and $\mathcal{H}_{1} \cap \mathcal{H}_{2}=\{0\}$, then the canonical dual fusion frames $\left(S_{\mathbf{W}, \mathbf{w}}^{-1} \mathbf{W}, \mathbf{v}\right)$ with arbitrary vector of weights $\mathbf{v}$ are probability $(1, \infty)$-loss optimal component preserving dual fusion frames of $(\mathbf{W}, \mathbf{w})$ but not the unique ones.

Proof. Let $A \in \mathfrak{L}_{T_{\mathbf{W}, \mathbf{w}}^{*}}$. Since $A T_{\mathbf{W}, \mathbf{w}}^{*}=S_{\mathbf{W}, \mathbf{w}}^{-1} T_{\mathbf{W}, \mathbf{w}} T_{\mathbf{W}, \mathbf{w}}^{*}=I_{\mathcal{H}}, T_{\mathbf{W}, \mathbf{w}}\left(A-S_{\mathbf{W}, \mathbf{w}}^{-1} T_{\mathbf{W}, \mathbf{w}}\right)^{*}=0$. By hypothesis this implies that $\left(\left(A-S_{\mathbf{W}, \mathbf{w}}^{-1} T_{\mathbf{W}, \mathbf{w}}\right)^{*} f\right)_{i}=0$ for each $i \in \Lambda_{1}$ and for each $f \in \mathcal{H}$, or equivalently, $M_{\Lambda_{1}}\left(A-S_{\mathbf{W}, \mathbf{w}}^{-1} T_{\mathbf{W}, \mathbf{w}}\right)^{*}=0$, i.e.,

$$
A M_{\Lambda_{1}}=S_{\mathbf{W}, \mathbf{w}}^{-1} T_{\mathbf{W}, \mathbf{w}} M_{\Lambda_{1}}
$$

By (3.2), (3.9) and (3.3),

$$
\begin{aligned}
\max _{1 \leq i \leq m}\left\|A \widehat{M}_{i} T_{\mathbf{W}, \mathbf{w}}^{*}\right\| & \geq \max _{i \in \Lambda_{1}}\left\|A \widehat{M}_{i} T_{\mathbf{W}, \mathbf{w}}^{*}\right\|=\max _{i \in \Lambda_{1}} q_{i} w_{i}\left\|A M_{i}\right\| \\
& =\max _{i \in \Lambda_{1}} q_{i} w_{i}\left\|S_{\mathbf{W}, \mathbf{w}}^{-1} T_{\mathbf{W}, \mathbf{w}} M_{i}\right\|=\max _{1 \leq i \leq m} q_{i} w_{i}\left\|S_{\mathbf{W}, \mathbf{w}}^{-1} T_{\mathbf{W}, \mathbf{w}} M_{i}\right\| \\
& =\max _{1 \leq i \leq m}\left\|S_{\mathbf{W}, \mathbf{w}}^{-1} T_{\mathbf{W}, \mathbf{w}} \widehat{M}_{i} T_{\mathbf{W}, \mathbf{w}}^{*}\right\| .
\end{aligned}
$$

Hence, $S_{\mathbf{W}, \mathbf{w}}^{-1} T_{\mathbf{W}, \mathbf{w}}$ is an element of the set (3.4).

Since $(\mathbf{W}, \mathbf{w})$ is not a Riesz fusion basis, there exists $A \in \mathfrak{L}_{T_{\mathbf{W}, \mathbf{w}}^{*}}$ such that $A \neq S_{\mathbf{W}, \mathbf{w}}^{-1} T_{\mathbf{W}, \mathbf{w}}$. By (3.9), $A M_{\Lambda_{2}} \neq S_{\mathbf{W}, \mathbf{w}}^{-1} T_{\mathbf{W}, \mathbf{w}} M_{\Lambda_{2}}$.

For $t \in \mathbb{R}, t \neq 0, S_{\mathbf{W}, \mathbf{w}}^{-1} T_{\mathbf{W}, \mathbf{w}}+t\left(A-S_{\mathbf{W}, \mathbf{w}}^{-1} T_{\mathbf{W}, \mathbf{w}}\right) \in \mathfrak{L}_{T_{\mathbf{W}, \mathbf{w}}^{*}}$. By (3.2), (3.9) and (3.3),

$$
\max _{i \in \Lambda_{1}}\left\|\left[S_{\mathbf{W}, \mathbf{w}}^{-1} T_{\mathbf{W}, \mathbf{w}}+t\left(A-S_{\mathbf{W}, \mathbf{w}}^{-1} T_{\mathbf{W}, \mathbf{w}}\right)\right] \widehat{M}_{i} T_{\mathbf{W}, \mathbf{w}}^{*}\right\|=c
$$

, and if $|t|>0$ is small enough, then

$$
\max _{i \in \Lambda_{2}}\left\|\left[S_{\mathbf{W}, \mathbf{w}}^{-1} T_{\mathbf{W}, \mathbf{w}}+t\left(A-S_{\mathbf{W}, \mathbf{w}}^{-1} T_{\mathbf{W}, \mathbf{w}}\right)\right] \widehat{M}_{i} T_{\mathbf{W}, \mathbf{w}}^{*}\right\|<c .
$$

Consequently, if $|t|>0$ is small enough, $S_{\mathbf{W}, \mathbf{w}}^{-1} T_{\mathbf{W}, \mathbf{w}}+t\left(A-S_{\mathbf{W}, \mathbf{w}}^{-1} T_{\mathbf{W}, \mathbf{w}}\right)$ is another element of the set (3.4).

Now the conclusion follows from Lemma 3.2.

Theorem 3.9. Let $(\mathbf{W}, \mathbf{w})$ be a fusion frame for $\mathcal{H}$ that is not a Riesz fusion basis. Let $c, \Lambda_{1}, \Lambda_{2}$ and $\mathcal{H}_{j}, j=1,2$ as in Theorem 3.3. If $\left|\Lambda_{1}\right|=1$ and $\left\{\left(W_{i}, w_{i}\right)\right\}_{i \in \Lambda_{2}}$ is a Riesz fusion basis for $\mathcal{H}_{2}$, then none of the canonical dual fusion frames $\left(S_{\mathbf{W}, \mathbf{w}}^{-1} \mathbf{W}, \mathbf{v}\right)$ with any vector of weights $\mathbf{v}$ is a probability $(1, \infty)$-loss optimal component preserving dual fusion frames of $(\mathbf{W}, \mathbf{w})$.

Proof. Without loss of generality suppose that $\Lambda_{1}=\{1\}$ and $\Lambda_{2}=\{2, \ldots, m\}$. Let $P: \mathcal{W} \rightarrow T_{\mathbf{W}, \mathbf{w}}^{*}(\mathcal{H})$ be the orthogonal projection onto $T_{\mathbf{W}, \mathbf{w}}^{*}(\mathcal{H})$. 
Let $B \in L(\mathcal{W}, \mathcal{H})$ such that $B\left(g_{i}\right)_{i=1}^{m}=g_{1}$ if $\left(g_{i}\right)_{i=1}^{m} \in\left(I_{\mathcal{W}}-P\right) M_{1} \mathcal{W}$ and $B\left(g_{i}\right)_{i=1}^{m}=0$ if $\left(g_{i}\right)_{i=1}^{m} \in$ $\left(\left(I_{\mathcal{W}}-P\right) M_{1} \mathcal{W}\right)^{\perp}$. Let $R:=B\left(I_{\mathcal{W}}-P\right)$.

Suppose that $\left(I_{\mathcal{W}}-P\right) M_{1} \mathcal{W}=\{0\}$, i.e., $M_{1} \mathcal{W} \subseteq T_{\mathbf{W}, \mathbf{w}}^{*}(\mathcal{H})$. Let $\left(f_{i}\right)_{i=1}^{m} \in \mathcal{W}$ such that $T_{\mathbf{W}, \mathbf{w}}\left(f_{i}\right)_{i=1}^{m}=$ 0 . By the previous inclusion there exists $f \in \mathcal{H}$ such that $M_{1}\left(f_{i}\right)_{i=1}^{m}=T_{\mathbf{W}, \mathbf{w}}^{*} f$. Consequently, $0=$ $\left\langle T_{\mathbf{W}, \mathbf{w}}\left(f_{i}\right)_{i=1}^{m}, f\right\rangle=\left\langle\left(f_{i}\right)_{i=1}^{m}, T_{\mathbf{W}, \mathbf{w}}^{*} f\right\rangle=\left\langle f_{1}, f_{1}\right\rangle$. Then $f_{1}=0$. Therefore, $0=T_{\mathbf{W}, \mathbf{w}}\left(f_{i}\right)_{i=1}^{m}=\sum_{i=2}^{m} w_{i} f_{i}$. Since $\left\{\left(W_{i}, w_{i}\right)\right\}_{i \in \Lambda_{2}}$ is a Riesz fusion basis for $\mathcal{H}_{2}$, this implies that $f_{i}=0$ for each $i=1, \ldots, m$. Taking into account the previous reasoning and that $(\mathbf{W}, \mathbf{w})$ is not a Riesz fusion basis for $\mathcal{H}$, there exists $\left(f_{i}\right)_{i=1}^{m} \in \mathcal{W}$ such that $\left(I_{\mathcal{W}}-P\right) M_{1}\left(f_{i}\right)_{i=1}^{m} \neq 0$, and hence, $R \neq 0$.

Since $R T_{\mathbf{W}, \mathbf{w}}^{*}=0, S_{\mathbf{W}, \mathbf{w}}^{-1} T_{\mathbf{W}, \mathbf{w}}+t R \in \mathfrak{L}_{T_{\mathbf{W}, \mathbf{w}}^{*}}$ for each $t \in \mathbb{R}$.

Let $\left\{e_{i, l}\right\}_{l \in L_{i}}$ be an orthonormal basis for $W_{i}$, for each $i=1, \ldots, m$. Then, $\left\{\left\{\left(\chi_{j}(i) e_{i, l}\right)_{i=1}^{m}\right\}_{l \in L_{j}}\right\}_{j=1}^{m}$ is an orthonormal basis for $\mathcal{W}$. Since

$$
\begin{aligned}
\operatorname{tr}\left(T_{\mathbf{W}, \mathbf{w}}^{*} S_{\mathbf{W}, \mathbf{w}}^{-1} R M_{1}\right) & =\sum_{j=1}^{m} \sum_{l_{j} \in L_{j}}\left\langle T_{\mathbf{W}, \mathbf{w}}^{*} S_{\mathbf{W}, \mathbf{w}}^{-1} R M_{1}\left(\chi_{j}(i) e_{i, l_{j}}\right)_{i=1}^{m},\left(\chi_{j}(i) e_{i, l_{j}}\right)_{i=1}^{m}\right\rangle \\
& =\sum_{l \in L_{1}}\left\langle T_{\mathbf{W}, \mathbf{w}}^{*} S_{\mathbf{W}, \mathbf{w}}^{-1} R M_{1}\left(\chi_{1}(i) e_{i, l}\right)_{i=1}^{m},\left(\chi_{1}(i) e_{i, l}\right)_{i=1}^{m}\right\rangle \\
& =\sum_{l \in L_{1}}\left\langle T_{\mathbf{W}, \mathbf{w}}^{*} S_{\mathbf{W}, \mathbf{w}}^{-1} e_{1, l},\left(\chi_{1}(i) e_{i, l}\right)_{i=1}^{m}\right\rangle \\
& =\sum_{l \in L_{1}}\left\langle S_{\mathbf{W}, \mathbf{w}}^{-1} e_{1, l}, w_{1} e_{1, l}\right\rangle=w_{1} \sum_{l \in L_{1}}\left\|S_{\mathbf{W}, \mathbf{w}}^{-1 / 2} e_{1, l}\right\|^{2}>0,
\end{aligned}
$$

using (3.2) and (3.3), if $t<0$ and $|t|$ is small enough,

$$
\begin{aligned}
& \left\|\left(S_{\mathbf{W}, \mathbf{w}}^{-1} T_{\mathbf{W}, \mathbf{w}}+t R\right) \widehat{M}_{1} T_{\mathbf{W}, \mathbf{w}}^{*}\right\|^{2}=w_{1}^{2} q_{1}^{2}\left\|\left(S_{\mathbf{W}, \mathbf{w}}^{-1} T_{\mathbf{W}, \mathbf{w}}+t R\right) M_{1}\right\|^{2} \\
& =w_{1}^{2} q_{1}^{2}\left\|S_{\mathbf{W}, \mathbf{w}}^{-1} T_{\mathbf{W}, \mathbf{w}} M_{1}\right\|^{2}+t^{2} w_{1}^{2} q_{1}^{2}\left\|R M_{1}\right\|^{2}+2 w_{1}^{2} q_{1}^{2} t \operatorname{Re}\left(\operatorname{tr}\left(T_{\mathbf{W}, \mathbf{w}}^{*} S_{\mathbf{W}, \mathbf{w}}^{-1} R M_{1}\right)\right)<c^{2}
\end{aligned}
$$

and $\left\|\left(S_{\mathbf{W}, \mathbf{w}}^{-1} T_{\mathbf{W}, \mathbf{w}}+t R\right) \widehat{M}_{i} T_{\mathbf{W}, \mathbf{w}}^{*}\right\| \leq w_{i} q_{i}\left\|S_{\mathbf{W}, \mathbf{w}}^{-1} T_{\mathbf{W}, \mathbf{w}} M_{i}\right\|+|t|\left\|R \widehat{M}_{i} T_{\mathbf{W}, \mathbf{w}}^{*}\right\|<c$ for each $i \in \Lambda_{2}$.

This shows that $S_{\mathbf{W}, \mathbf{w}}^{-1} T_{\mathbf{W}, \mathbf{w}}$ is not an element of the set (3.4). By Lemma 3.2, we conclude that none of the canonical dual fusion frames $\left(S_{\mathbf{W}, \mathbf{w}}^{-1} \mathbf{W}, \mathbf{v}\right)$ with any vector of weights $\mathbf{v}$ is a probability $(1, \infty)$-loss optimal component preserving dual fusion frame of $(\mathbf{W}, \mathbf{w})$.

TheOREm 3.10. Let $(\mathbf{W}, \mathbf{w})$ be a fusion frame for $\mathcal{H}$ that is not a Riesz fusion basis. Let $c, \Lambda_{j}$ and $\mathcal{H}_{j}$, $j=1,2$, as in Theorem 3.3. If $\left\{\left(W_{i}, w_{i}\right)\right\}_{i \in \Lambda_{1}}$ is a Riesz fusion basis for $\mathcal{H}_{1}$ and there exists $\left(f_{j}\right)_{j=1}^{m} \in \mathcal{W}$ such that $f_{j} \neq 0$ for each $j \in \Lambda_{1}$ and $T_{\mathbf{W}, \mathbf{w}}\left(f_{j}\right)_{j=1}^{m}=0$, then none of the canonical dual fusion frames $\left(S_{\mathbf{W}, \mathbf{w}}^{-1} \mathbf{W}, \mathbf{v}\right)$ with any vector of weights $\mathbf{v}$ is a probability $(1, \infty)$-loss optimal component preserving dual fusion frames of $(\mathbf{W}, \mathbf{w})$.

Proof. The proof is similar to that of Proposition 3.7, so we only sketch it. Let $\left\{e_{i, l}\right\}_{l \in L_{i}}$ be an orthonormal basis for $W_{i}$ for each $i=1, \ldots, m$. Then there exists $f \in \mathcal{H}$ such that $\left\langle f, S_{\mathbf{W}, \mathbf{w}}^{-1} e_{i, l}\right\rangle=-\left\langle f_{i}, e_{i, l}\right\rangle$ for each $i \in \Lambda_{1}$ and $l \in L_{i}$.

Let $R \in L(\mathcal{W}, \mathcal{H})$, given by $R\left(g_{j}\right)_{j=1}^{m}=\left\langle\left(g_{j}\right)_{j=1}^{m},\left(f_{j}\right)_{j=1}^{m}\right\rangle f$ for each $\left(g_{j}\right)_{j=1}^{m} \in \mathcal{W}$. Since $T_{\mathbf{W}, \mathbf{w}} R^{*}=0$, then $S_{\mathbf{W}, \mathbf{w}}^{-1} T_{\mathbf{W}, \mathbf{w}}+t R \in \mathfrak{L}_{T_{\mathbf{W}, \mathbf{w}}^{*}}$ for each $t \in \mathbb{R}$. 
If $k \in \Lambda_{1}, \operatorname{tr}\left(T_{\mathbf{W}, \mathbf{w}}^{*} S_{\mathbf{W}, \mathbf{w}}^{-1} R M_{k}\right)=-w_{k}\left\|f_{k}\right\|^{2}<0$. Hence, if $t>0$ is small enough, $\|\left(S_{\mathbf{W}, \mathbf{w}}^{-1} T_{\mathbf{W}, \mathbf{w}}+\right.$ $t R) \widehat{M}_{k} \|^{2}<c^{2}$ for each $k \in \Lambda_{1}$ and $\left\|\left(S_{\mathbf{W}, \mathbf{w}}^{-1} T_{\mathbf{W}, \mathbf{w}}+t R\right) \widehat{M}_{k}\right\|<c$ for each $k \in \Lambda_{2}$. This shows that $S_{\mathbf{W}, \mathbf{w}}^{-1} T_{\mathbf{W}, \mathbf{w}}$ is not an element of the set (3.4). By Lemma 3.2, this says that none of the canonical dual fusion frames $\left(S_{\mathbf{W}, \mathbf{w}}^{-1} \mathbf{W}, \mathbf{v}\right)$ with any vector of weights $\mathbf{v}$ is a probability $(1, \infty)$-loss optimal component preserving dual fusion frames of $(\mathbf{W}, \mathbf{w})$.

4. Probability optimal duals in relation with general optimal duals. A greater probability $p_{i}$, and then a greater number $q_{i}$, implies that the subspace $W_{i}$ is more probable of being erased. In this section, we analyze situations in which if the subspaces with greater probabilities are erased, then the actual error for the probability optimal dual is less than or equal to the error for the general optimal dual. This shows the convenience of using probability optimal dual fusion frames to obtain a better reconstruction in these situations.

Proposition 4.1. Let $((\widehat{\mathbf{V}}, \widehat{\mathbf{v}}), \widehat{Q}) \in \widehat{\mathcal{D}}_{1}^{(\infty)}(\mathbf{W}, \mathbf{w})$ and $((\mathbf{V}, \mathbf{v}), Q) \in \mathcal{D}_{1}^{(\infty)}(\mathbf{W}, \mathbf{w})$. If $q_{i} \leq q_{1}$ and $\left\|T_{\mathbf{V}, \mathbf{v}} Q M_{i} T_{\mathbf{W}, \mathbf{w}}^{*}\right\| \leq\left\|T_{\mathbf{V}, \mathbf{v}} Q M_{1} T_{\mathbf{W}, \mathbf{w}}^{*}\right\|$ for each $i=2, \ldots, m$, then $\left\|T_{\widehat{\mathbf{v}}, \widehat{\mathbf{v}}} \widehat{Q} M_{1} T_{\mathbf{W}, \mathbf{w}}^{*}\right\| \leq\left\|T_{\mathbf{V}, \mathbf{v}} Q M_{1} T_{\mathbf{W}, \mathbf{w}}^{*}\right\|$.

Proof. By the definitions of $\mathcal{D}_{1}^{(\infty)}(\mathbf{W}, \mathbf{w})$ and $\widehat{\mathcal{D}}_{1}^{(\infty)}(\mathbf{W}, \mathbf{w})$,

$$
\begin{aligned}
& \left\|T_{\widehat{\mathbf{V}}, \widehat{\mathbf{v}}} \widehat{Q} M_{1} T_{\mathbf{W}, \mathbf{w}}^{*}\right\| \leq \max _{1 \leq i \leq m} \frac{q_{i}}{q_{1}}\left\|T_{\widehat{\mathbf{V}}, \widehat{\mathbf{v}}} \widehat{Q} M_{i} T_{\mathbf{W}, \mathbf{w}}^{*}\right\|=\min _{B \in \mathfrak{L}_{T_{\mathbf{W}, \mathbf{w}}}} \max _{1 \leq i \leq m} \frac{q_{i}}{q_{1}}\left\|B M_{i} T_{\mathbf{W}, \mathbf{w}}^{*}\right\| \\
& \leq \min _{B \in \mathfrak{L}_{T_{\mathbf{W}, \mathbf{w}}}^{*}} \max _{1 \leq i \leq m}\left\|B M_{i} T_{\mathbf{W}, \mathbf{w}}^{*}\right\|=\max _{1 \leq i \leq m}\left\|T_{\mathbf{V}, \mathbf{v}} Q M_{i} T_{\mathbf{W}, \mathbf{w}}^{*}\right\|=\left\|T_{\mathbf{V}, \mathbf{v}} Q M_{1} T_{\mathbf{W}, \mathbf{w}}^{*}\right\| .
\end{aligned}
$$

Proposition 4.2. Let $((\widehat{\mathbf{V}}, \widehat{\mathbf{v}}), \widehat{Q}) \in \widehat{\mathcal{D}}_{1}^{(\infty)}(\mathbf{W}, \mathbf{w})$ and $(\mathbf{V}, \mathbf{v})$ be a $Q$-component preserving dual fusion frame of $(\mathbf{W}, \mathbf{w})$. If $q_{i} \leq q_{1}$ and $q_{i}\left\|T_{\mathbf{V}, \mathbf{v}} Q M_{i} T_{\mathbf{W}, \mathbf{w}}^{*}\right\| \leq q_{1}\left\|T_{\mathbf{V}, \mathbf{v}} Q M_{1} T_{\mathbf{W}, \mathbf{w}}^{*}\right\|$ for each $i=2, \ldots, m$, then $\left\|T_{\widehat{\mathbf{V}}, \widehat{\mathbf{v}}} \widehat{Q} M_{1} T_{\mathbf{W}, \mathbf{w}}^{*}\right\| \leq\left\|T_{\mathbf{V}, \mathbf{v}} Q M_{1} T_{\mathbf{W}, \mathbf{w}}^{*}\right\|$. If we also have $((\mathbf{V}, \mathbf{v}), Q) \notin \widehat{\mathcal{D}}_{1}^{(\infty)}(\mathbf{W}, \mathbf{w})$, then $\left\|T_{\widehat{\mathbf{V}}, \widehat{\mathbf{v}}} \widehat{Q} M_{1} T_{\mathbf{W}, \mathbf{w}}^{*}\right\|$ $<\left\|T_{\mathbf{V}, \mathbf{v}} Q M_{1} T_{\mathbf{W}, \mathbf{w}}^{*}\right\|$.

Proof. By the definition of $\widehat{\mathcal{D}}_{1}^{(\infty)}(\mathbf{W}, \mathbf{w})$,

$$
\begin{aligned}
q_{1}\left\|T_{\widehat{\mathbf{V}}, \widehat{\mathbf{v}}} \widehat{Q} M_{1} T_{\mathbf{W}, \mathbf{w}}^{*}\right\| & \leq \max _{1 \leq i \leq m} q_{i}\left\|T_{\widehat{\mathbf{V}}, \widehat{\mathbf{v}}} \widehat{Q} M_{i} T_{\mathbf{W}, \mathbf{w}}^{*}\right\|=\min _{B \in \mathfrak{L}_{T, \mathbf{W}, \mathbf{w}}^{*}} \max _{1 \leq i \leq m} q_{i}\left\|B M_{i} T_{\mathbf{W}, \mathbf{w}}^{*}\right\| \\
& \leq \max _{1 \leq i \leq m} q_{i}\left\|T_{\mathbf{V}, \mathbf{v}} Q M_{i} T_{\mathbf{W}, \mathbf{w}}^{*}\right\|=q_{1}\left\|T_{\mathbf{V}, \mathbf{v}} Q M_{1} T_{\mathbf{W}, \mathbf{w}}^{*}\right\|
\end{aligned}
$$

and hence, $\left\|T_{\widehat{\mathbf{V}}, \widehat{\mathbf{v}}} \widehat{Q} M_{1} T_{\mathbf{W}, \mathbf{w}}^{*}\right\| \leq\left\|T_{\mathbf{V}, \mathbf{v}} Q M_{1} T_{\mathbf{W}, \mathbf{w}}^{*}\right\|$. If $((\mathbf{V}, \mathbf{v}), Q) \notin \widehat{\mathcal{D}}_{1}^{(\infty)}(\mathbf{W}, \mathbf{w})$, the above second inequality is strict and then $\left\|T_{\widehat{\mathbf{V}}, \widehat{\mathbf{v}}} \widehat{Q} M_{1} T_{\mathbf{W}, \mathbf{w}}^{*}\right\|<\left\|T_{\mathbf{V}, \mathbf{v}} Q M_{1} T_{\mathbf{W}, \mathbf{w}}^{*}\right\|$.

The following corollary follows immediately from Proposition 4.2.

COROLlary 4.3. Let $((\widehat{\mathbf{V}}, \widehat{\mathbf{v}}), \widehat{Q}) \in \widehat{\mathcal{D}}_{1}^{(\infty)}(\mathbf{W}, \mathbf{w})$ and $(\mathbf{V}, \mathbf{v})$ be a $Q$-component preserving dual fusion frame of $(\mathbf{W}, \mathbf{w})$. If $q_{i} \leq q_{1}$ and $\left\|T_{\mathbf{V}, \mathbf{v}} Q M_{1} T_{\mathbf{W}, \mathbf{w}}^{*}\right\|=\cdots=\left\|T_{\mathbf{V}, \mathbf{v}} Q M_{m} T_{\mathbf{W}, \mathbf{w}}^{*}\right\|$, then

$$
\left\|T_{\widehat{\mathbf{v}}, \widehat{\mathbf{v}}} \widehat{Q} M_{1} T_{\mathbf{W}, \mathbf{w}}^{*}\right\| \leq\left\|T_{\mathbf{V}, \mathbf{v}} Q M_{1} T_{\mathbf{W}, \mathbf{w}}^{*}\right\| .
$$

Moreover, if we also have $((\mathbf{V}, \mathbf{v}), Q) \notin \widehat{\mathcal{D}}_{1}^{(\infty)}(\mathbf{W}, \mathbf{w})$, then

$$
\left\|T_{\widehat{\mathbf{V}}, \widehat{\mathbf{v}}} \widehat{Q} M_{1} T_{\mathbf{W}, \mathbf{w}}^{*}\right\|<\left\|T_{\mathbf{V}, \mathbf{v}} Q M_{1} T_{\mathbf{W}, \mathbf{w}}^{*}\right\| .
$$


Proposition 4.4. Let $((\widehat{\mathbf{V}}, \widehat{\mathbf{v}}), \widehat{Q}) \in \widehat{\mathcal{D}}_{1}^{(\infty)}(\mathbf{W}, \mathbf{w})$ and $((\mathbf{V}, \mathbf{v}), Q) \in \mathcal{D}_{1}^{(\infty)}(\mathbf{W}, \mathbf{w}) \backslash \widehat{\mathcal{D}}_{1}^{(\infty)}(\mathbf{W}, \mathbf{w})$. If $q_{1}>q_{2}=\cdots=q_{m}$ and $\left\|T_{\widehat{\mathbf{V}}, \widehat{\mathbf{v}}} \widehat{Q} \widehat{M}_{1} T_{\mathbf{W}, \mathbf{w}}^{*}\right\|=\cdots=\left\|T_{\widehat{\mathbf{v}}, \widehat{\mathbf{v}}} \widehat{Q} \widehat{M}_{m} T_{\mathbf{W}, \mathbf{w}}^{*}\right\|$, then

$$
\left\|T_{\widehat{\mathbf{V}}, \widehat{\mathbf{v}}} \widehat{Q} M_{1} T_{\mathbf{W}, \mathbf{w}}^{*}\right\|<\left\|T_{\mathbf{V}, \mathbf{v}} Q M_{1} T_{\mathbf{W}, \mathbf{w}}^{*}\right\|
$$

Proof. We have

$$
\begin{gathered}
\left\|T_{\widehat{\mathbf{v}}, \widehat{\mathbf{v}}} \widehat{Q} M_{1} T_{\mathbf{W}, \mathbf{w}}^{*}\right\|<\left\|T_{\widehat{\mathbf{v}}, \widehat{\mathbf{v}}} \widehat{Q} M_{2} T_{\mathbf{W}, \mathbf{w}}^{*}\right\|=\cdots=\left\|T_{\widehat{\mathbf{v}}, \widehat{\mathbf{v}}} \widehat{Q} M_{m} T_{\mathbf{W}, \mathbf{w}}^{*}\right\|, \\
q_{j}\left\|T_{\widehat{\mathbf{v}}, \widehat{\mathbf{v}}} \widehat{Q} M_{j} T_{\mathbf{W}, \mathbf{w}}^{*}\right\|<\max _{1 \leq i \leq m} q_{i}\left\|T_{\mathbf{V}, \mathbf{v}} Q M_{i} T_{\mathbf{W}, \mathbf{w}}^{*}\right\|, \quad \forall j \in\{1, \ldots, m\},
\end{gathered}
$$

and

$$
\left\|T_{\mathbf{V}, \mathbf{v}} Q M_{j} T_{\mathbf{W}, \mathbf{w}}^{*}\right\| \leq \max _{1 \leq i \leq m}\left\|T_{\widehat{\mathbf{v}}, \widehat{\mathbf{v}}} \widehat{Q} M_{i} T_{\mathbf{W}, \mathbf{w}}^{*}\right\|, \quad \forall j \in\{1, \ldots, m\} .
$$

By (4.10) and (4.12), $q_{i}\left\|T_{\mathbf{V}, \mathbf{v}} Q M_{i} T_{\mathbf{W}, \mathbf{w}}^{*}\right\| \leq q_{i}\left\|T_{\widehat{\mathbf{V}}, \widehat{\mathbf{v}}} \widehat{Q} M_{i} T_{\mathbf{W}, \mathbf{w}}^{*}\right\|$ for each $i=2, \ldots, m$. Therefore, by (4.11), $\max _{1 \leq i \leq m} q_{i}\left\|T_{\mathbf{V}, \mathbf{v}} Q M_{i} T_{\mathbf{W}, \mathbf{w}}^{*}\right\|=q_{1}\left\|T_{\mathbf{V}, \mathbf{v}} Q M_{1} T_{\mathbf{W}, \mathbf{w}}^{*}\right\|$ and hence, $\left\|T_{\widehat{\mathbf{V}}, \widehat{\mathbf{v}}} \widehat{Q} M_{1} T_{\mathbf{W}, \mathbf{w}}^{*}\right\|<\left\|T_{\mathbf{V}, \mathbf{v}} Q M_{1} T_{\mathbf{W}, \mathbf{w}}^{*}\right\|$.

Now we present two illustrative examples.

EXAmple 4.5. Consider the subspaces of $\mathbb{R}^{3}, W_{1}=\{(1,0,0)\}^{\perp}$ and $W_{2}=\{(0,1,0)\}^{\perp}$ and arbitrary weigths $w_{1}>0$ and $w_{2}>0$. Then $(\mathbf{W}, \mathbf{w})$ is a fusion frame for $\mathbb{R}^{3}$ with $S_{\mathbf{W}, \mathbf{w}}^{-1} \mathbf{W}=\mathbf{W}$. This fusion frame was considered in [8, Example 6.3]. It is easy to see that $\left\|S_{\mathbf{W}, \mathbf{w}}^{-1} \pi_{W_{i}}\right\|^{2}=\frac{1}{w_{i}^{4}}+\frac{1}{\left(w_{1}^{2}+w_{2}^{2}\right)^{2}}$ for $i=1,2$.

Suppose that $0<p_{2}<p_{1}<1$ and $\beta:=\frac{p_{2}}{p_{1}} \geq \frac{\sqrt{2}}{2}$. This implies that $\frac{1}{2}<p_{1}<2-\sqrt{2}$ and $\sqrt{2}-1<p_{2}<\frac{1}{2}$. Let $q_{1}=\alpha p_{1}, q_{2}=\alpha p_{2}$ for certain $\alpha>0$.

We have, $q_{1} w_{1}^{2}\left\|S_{\mathbf{W}, \mathbf{w}}^{-1} \pi_{W_{1}}\right\|=q_{2} w_{2}^{2}\left\|S_{\mathbf{W}, \mathbf{w}}^{-1} \pi_{W_{2}}\right\|$ if and only if $\beta=\sqrt{\frac{w_{1}^{4}+\left(w_{1}^{2}+w_{2}^{2}\right)^{2}}{w_{2}^{4}+\left(w_{1}^{2}+w_{2}^{2}\right)^{2}}}$. Consequently, $w_{1}^{2}=w_{2}^{2} \frac{\left(\beta^{2}-1\right)+\sqrt{\left(1-\beta^{2}\right)^{2}-\left(2-\beta^{2}\right)\left(1-2 \beta^{2}\right)}}{2-\beta^{2}}$. By [8, Example 6.3], $(\mathbf{W}, \mathbf{v})$ is a general $(1, \infty)$-loss optimal $Q$-component preserving dual fusion frame of $(\mathbf{W}, \mathbf{w})$, where $Q: \mathcal{W} \rightarrow \mathcal{V}, Q\left(\left(0, x_{2}, x_{3}\right),\left(y_{1}, 0, y_{3}\right)\right)=$ $\left(\frac{1}{v_{1}}\left(0, \frac{x_{2}}{w_{1}}, \frac{x_{3}}{2 w_{1}}\right), \frac{1}{v_{2}}\left(\frac{y_{1}}{w_{2}}, 0, \frac{y_{3}}{2 w_{2}}\right)\right)$. Since $w_{1} \neq w_{2}$, then $Q \neq Q_{S_{\mathbf{W}, \mathbf{w}}^{-1} T_{\mathbf{W}, \mathbf{w}}, \mathbf{v}}$ and $T_{\mathbf{W}, \mathbf{v}} Q \neq T_{\mathbf{W}, \mathbf{v}} Q_{S_{\mathbf{W}, \mathbf{w}}^{-1} T_{\mathbf{W}, \mathbf{w}}, \mathbf{v}}$. So, by Proposition 4.4,

$$
\left\|T_{\mathbf{W}, \mathbf{v}} Q_{S_{\mathbf{W}, \mathbf{w}}^{-1} T_{\mathbf{W}, \mathbf{w}}, \mathbf{v}} M_{1} T_{\mathbf{W}, \mathbf{w}}^{*}\right\|<\left\|T_{\mathbf{W}, \mathbf{v}} Q M_{1} T_{\mathbf{W}, \mathbf{w}}^{*}\right\| .
$$

Indeed, $\left\|T_{\mathbf{W}, \mathbf{v}} Q_{S_{\mathbf{W}, \mathbf{w}}^{-1} T_{\mathbf{W}, \mathbf{w}}, \mathbf{v}} M_{1} T_{\mathbf{W}, \mathbf{w}}^{*}\right\|^{2}=1+\left(\frac{w_{1}^{2}}{w_{1}^{2}+w_{2}}\right)^{2}$ and $\left\|T_{\mathbf{W}, \mathbf{v}} Q M_{1} T_{\mathbf{W}, \mathbf{w}}^{*}\right\|^{2}=\frac{5}{4}$.

ExAmple 4.6. Let $\mathcal{H}=\mathbb{R}^{3}, W_{1}=\{(1,0,0)\}^{\perp}, W_{2}=\{(0,1,0)\}^{\perp}, W_{3}=\{(0,0,1)\}^{\perp}, w_{1}>0, w_{2}>0$ and $w_{3}>0$. Then $(\mathbf{W}, \mathbf{w})$ is a fusion frame for $\mathbb{R}^{3}, S_{\mathbf{W}, \mathbf{w}}^{-1}\left(x_{1}, x_{2}, x_{3}\right)=\left(\frac{x_{1}}{w_{2}^{2}+w_{3}^{2}}, \frac{x_{2}}{w_{1}^{2}+w_{3}^{2}}, \frac{x_{3}}{w_{1}^{2}+w_{2}^{2}}\right)$ and $S_{\mathbf{W}, \mathbf{w}}^{-1} \mathbf{W}=\mathbf{W}$.

Set $w_{1}=w_{2}=w_{3}=w$. By Corollary 3.4 (with $q_{1}=q_{2}=q_{2}=1$ ), since $w^{2}\left\|S_{\mathbf{W}, w}^{-1} \pi_{W_{1}}\right\|=$ $w^{2}\left\|S_{\mathbf{W}, w}^{-1} \pi_{W_{2}}\right\|=w^{2}\left\|S_{\mathbf{W}, w}^{-1} \pi_{W_{3}}\right\|$, the only general $(r, \infty)$-loss optimal component preserving dual fusion frames of $(\mathbf{W}, w)$ are the canonical ones $(\mathbf{W}, \mathbf{v})$ with arbitrary vector of weights $\mathbf{v}$.

By Theorem 3.10, if $q_{1}>q_{2}$ and $q_{1}>q_{3}$, then none of the canonical dual fusion frames $(\mathbf{W}, \mathbf{v})$ with any vector of weights $\mathbf{v}$ is a probability $(1, \infty)$-loss optimal component preserving dual fusion frames of $(\mathbf{W}, w)$. By Corollary 4.3 , if $((\widehat{\mathbf{V}}, \widehat{\mathbf{v}}), \widehat{Q}) \in \widehat{\mathcal{D}}_{1}^{(\infty)}(\mathbf{W}, w)$ then $\left\|T_{\widehat{\mathbf{V}}, \widehat{\mathbf{v}}} \widehat{Q} M_{1} T_{\mathbf{W}, w}^{*}\right\|<\left\|T_{\mathbf{W}, \mathbf{v}} Q_{S_{\mathbf{W}, w}^{-1} T_{\mathbf{W}, w}, \mathbf{v}} M_{1} T_{\mathbf{W}, w}^{*}\right\|$. We can see this explicitly as follows. 
For $q_{1}>0, q_{2}>0$ and $q_{3}>0$, the unique element in the set (3.4) is given by

$$
A\left(\left(0, x_{2}, x_{3}\right),\left(y_{1}, 0, y_{3}\right),\left(z_{1}, z_{2}, 0\right)\right)=\frac{1}{w}\left(\frac{\sqrt{q_{3}} y_{1}+\sqrt{q_{2}} z_{1}}{\sqrt{q_{2}}+\sqrt{q_{3}}}, \frac{\sqrt{q_{3}} x_{2}+\sqrt{q_{1}} z_{2}}{\sqrt{q_{1}}+\sqrt{q_{3}}}, \frac{\sqrt{q_{2}} x_{3}+\sqrt{q_{1}} y_{3}}{\sqrt{q_{1}}+\sqrt{q_{2}}}\right) .
$$

We have $A M_{i} \mathcal{W}=W_{i}$ for each $i=1,2,3$ and

$$
\begin{aligned}
& Q_{A, \mathbf{v}}\left(\left(0, x_{2}, x_{3}\right),\left(y_{1}, 0, y_{3}\right),\left(z_{1}, z_{2}, 0\right)\right) \\
& =\frac{1}{w}\left(\frac{1}{v_{1}}\left(0, \frac{\sqrt{q_{3}} x_{2}}{\sqrt{q_{1}}+\sqrt{q_{3}}}, \frac{\sqrt{q_{2}} x_{3}}{\sqrt{q_{1}}+\sqrt{q_{2}}}\right), \frac{1}{v_{2}}\left(\frac{\sqrt{q_{3}} y_{1}}{\sqrt{q_{2}}+\sqrt{q_{3}}}, 0, \frac{\sqrt{q_{1}} y_{3}}{\sqrt{q_{1}}+\sqrt{q_{2}}}\right), \frac{1}{v_{3}}\left(\frac{\sqrt{q_{2}} z_{1}}{\sqrt{q_{2}}+\sqrt{q_{3}}}, \frac{\sqrt{q_{1}} z_{2}}{\sqrt{q_{1}}+\sqrt{q_{3}}}, 0\right)\right) .
\end{aligned}
$$

With this $A$ we obtain that $(\mathbf{W}, \mathbf{v})$ is the unique (up to weights) probability $\left(r, \infty\right.$ )-loss optimal $Q_{A, \mathbf{v}^{-}}$ component preserving dual fusion frame of $(\mathbf{W}, w)$. It is easy to see that $\left\|T_{\mathbf{W}, \mathbf{v}} Q_{S_{\mathbf{W}, w}^{-1} T_{\mathbf{W}, w}, \mathbf{v}} M_{1} T_{\mathbf{W}, w}^{*}\right\|^{2}=\frac{1}{2}$ and $\left\|T_{\mathbf{W}, \mathbf{v}} Q_{A, \mathbf{v}} M_{1} T_{\mathbf{W}, w}^{*}\right\|^{2}=\frac{q_{3}}{\left(\sqrt{q_{1}}+\sqrt{q_{3}}\right)^{2}}+\frac{q_{2}}{\left(\sqrt{q_{1}}+\sqrt{q_{2}}\right)^{2}}$.

If the numbers $q_{i}$ for $i=1,2,3$, are not all equal, $A \neq S_{\mathbf{W}, w}^{-1} T_{\mathbf{W}, w}$. If $q_{1}>q_{2}$ and $q_{1}>q_{3}$, then $\left\|T_{\mathbf{W}, \mathbf{v}} Q_{A, \mathbf{v}} M_{1} T_{\mathbf{W}, w}^{*}\right\|^{2}<\left\|T_{\mathbf{W}, \mathbf{v}} Q_{S_{\mathbf{W}, w}^{-1} T_{\mathbf{W}, w}, \mathbf{v}} M_{1} T_{\mathbf{W}, w}^{*}\right\|^{2}$. Moreover, if $q_{1}>q_{2}>q_{3}$, then $W_{1}$ and $W_{2}$ are more probable of being erased than $W_{3}$, and since $\left\|T_{\mathbf{W}, \mathbf{v}} Q_{S_{\mathbf{W}, w}^{-1} T \mathbf{W}, w, \mathbf{v}} M_{\{1,2\}} T_{\mathbf{W}, w}^{*}\right\|^{2}=\frac{3}{2}$ and $\left\|T_{\mathbf{W}, \mathbf{v}} Q_{A, \mathbf{v}} M_{\{1,2\}} T_{\mathbf{W}, w}^{*}\right\|^{2}=\frac{q_{3}}{\left(\sqrt{q_{2}}+\sqrt{q_{3}}\right)^{2}}+\frac{q_{1}+q_{2}}{\left(\sqrt{q_{1}}+\sqrt{q_{2}}\right)^{2}}+\frac{q_{3}}{\left(\sqrt{q_{1}}+\sqrt{q_{3}}\right)^{2}}$, it follows

$$
\left\|T_{\mathbf{W}, \mathbf{v}} Q_{A, \mathbf{v}} M_{\{1,2\}} T_{\mathbf{W}, w}^{*}\right\|^{2}<\left\|T_{\mathbf{W}, \mathbf{v}} Q_{S_{\mathbf{W}, w}^{-1} T_{\mathbf{W}, w}, \mathbf{v}} M_{\{1,2\}} T_{\mathbf{W}, w}^{*}\right\|^{2} .
$$

Acknowledgment. The author thanks the valuable comments and suggestions of the referee and of the Handling Editor, Professor Zejun Huang, that improved the presentation of the paper.

\section{REFERENCES}

[1] B.G. Bodmann. Optimal linear transmission by loss-insensitive packet encoding. Appl. Comput. Harmon. Anal., 22:274$285,2007$.

[2] P.G. Casazza and G. Kutyniok (editors). Finite Frames. Theory and Applications. Birkhäuser, Boston, 2012.

[3] P.G. Casazza and G. Kutyniok. Frames of subspaces. Contemp. Math., 345:87-113, 2004.

[4] P.G. Casazza and G. Kutyniok. Robustness of fusion frames under erasures of subspaces and of local frame vectors. In: E.L. Grinberg, D. Larson, P.E.T. Jorgensen, P. Massopust, G. Olafsson, E.T. Quinto, and B. Rubi (editors), Radon Transforms, Geometry and Wavelets, Contemp. Math., 464:149-160, 2008.

[5] P.G. Casazza, G. Kutyniok, and S. Li. Fusion frames and distributed processing. Appl. Comput. Harmon. Anal., 25:114-132, 2008.

[6] O. Christensen. An Introduction to Frames and Riesz Bases. Birkhäuser, Boston, 2003.

[7] S.B. Heineken, P.M. Morillas, A.M. Benavente, and M.I. Zakowicz. Dual fusion frames. Arch. Math., 103:355-365, 2014.

[8] S.B. Heineken and P.M. Morillas. Properties of finite dual fusion frames. Linear Algebra Appl., 453:1-27, 2014.

[9] J. Leng and D. Han. Optimal dual frames for erasures II. Linear Algebra Appl., 435:1464-1472, 2011.

[10] J. Leng, D. Han, and T. Huang. Optimal dual frames for comunication coding with probabilistic erasures. IEEE Trans. Signal Process, 59:5380-5389, 2011.

[11] J. Leng, D. Han, and T. Huang. Probability modelled optimal frames for erasures. Linear Algebra Appl., 438:4222-4236, 2013.

[12] J. Lopez and D. Han. Optimal dual frames for erasures. Linear Algebra Appl., 432:471-482, 2010.

[13] J. Kovačević and A. Chebira. An introduction to frames. Found. Trends Signal Process., 2:1-94, 2008.

[14] G. Kutyniok, A. Pezeshki, A.R. Calderbank, and T. Liu. Robust dimension reduction, fusion frames, and Grassmannian packings. Appl. Comput. Harmon. Anal., 26:64-76, 2009.

[15] P.G. Massey, M.A. Ruiz, and D. Stojanoff. Robust dual reconstruction systems and fusion frames. Acta Appl. Math., 119:167-183, 2012.

[16] P.M. Morillas. Group reconstruction systems. Electron. J. Linear Algebra, 22:875-911, 2011.

[17] P.M. Morillas. Harmonic reconstruction systems. Electron. J. Linear Algebra, 26:692-705, 2013. 